\title{
Exploring state factor landscapes
}

\author{
K. DANA CHADWICK ${ }^{1}$
}

${ }^{1}$ Department of Earth System Science, Stanford University, 365 Panama St., Stanford, CA. kdc@stanford.edu

The state factor framework has been fundamental to our understanding of how soils and ecosystems develop, as well as what we expect their characteristics to be in new locations. New remote sensing data provide an opportunity for exploring biogeochemical, ecological, and geomorphic gradients that expand our understanding of the influence state factors play in soil and ecosystem development. High resolution lidar data provide insights into topography and landscape evolution processes that control the underlying template on which soils develop. Even when it is not possible to see through the vegetation to the soil surface, imaging spectroscopy data can now capture information on the nutrient status of vegetation at the scale of individuals that informs our understanding of soil biogeochemistry across space. In my work I have utilized high spatial resolution remote sensing datasets to integrate observed vegetation patterns with soil biogeochemical analysis and landscape evolution modeling in order to develop an understanding of how geomorphic and biogeochemical processes act to organize ecosystems. Here I draw from several studies to offer my perspective on the role of these high resolution datasets in furthering our understanding of the state factor framework, consider how these data can help us to better understand soil biogeochemical landscapes, and reflect on how explorations of these factors have shaped me as a member of the scientific community. 